\title{
Can we detect polygenic selection on cognitive ability using GWAS hits? Employing random SNPs as a null model.
}

\section{Davide Piffer}

Department of Psychology, Ben Gurion University of the Negev, Beer Sheva, Israel; Email: piffer@post.bgu.ac.il

\begin{abstract}
Background: The genetic variants identified by three large genome-wide association studies (GWAS) of educational attainment were used to test a polygenic selection model.

ethods: Average frequencies of alleles with positive effect (polygenic scores or PS) were compared across populations $(\mathrm{N}=26)$ using data from 1000 Genomes. A null model was created using frequencies of random SNPs.

Results: Polygenic selection signal of educational attainment GWAS hits is high among a handful of SNPs within genomic regions replicated across GWAS publications. A polygenic score comprising 9 SNPs predicts population IQ ( $r=0.9)$, outperforming $99.9 \%$ of the polygenic scores obtained from sets of random SNPs. Its predictive power remains unaffected after controlling for spatial autocorrelation. Even random polygenic scores are moderate predictors of population IQ (thanks to spatial autocorrelation), and their predictive power increases logarithmically with the number of SNPs, indicating an exponential reduction in noise.

Conclusion: This study provides guidance for using GWAS hits together with random SNPs for testing polygenic selection.
\end{abstract}

\section{Introduction}

Over the last decade, population geneticists have recognized that most traits are highly polygenic, and hence have moved away from the study of genetic evolution using the single-gene, Mendelian approach, towards models that examine many genes together (i.e. polygenic models).

Signals of polygenic selection can be identified by various methods, such as correlation of allele frequencies [1-4] and the regression of average trait values on polygenic scores (PS) [2,5-7], which have been successfully applied to human stature [5-7] and cognitive abilities [2]. This paper has several aims: to test the presence of correlated frequencies among GWAS hits and the predictive power of polygenic scores (average frequencies of GWAS alleles with positive effect), independently of spatial autocorrelation. A null model will be built using a large set of random SNPs and the polygenic selection model will be tested against it. Piffer [8] identified 9 genomic loci (table S1) that were replicated across the three largest GWAS of educational attainment published to date [9-11]. The 9 loci contain GWAS significant alleles that were found to be in strong LD ( $r>0.8)$. One locus was replicated across three GWAS [9-11] and the same SNP (rs9320913) was found in two of them[9,11]. The population frequencies of the 9 pairs (one member belonging to each GWAS publication) of alleles were highly correlated $(r=0.919)$, hence the SNPs published in [4] were used. Thus, this set of 9 SNPs was considered the best candidate for analysis of natural selection on educational attainment and related phenotypes (e.g. general cognitive ability or gca). Another set of 7 SNPs that reached significance in the UK Biobank and another 
database was identified by [11]. In addition, the full set of 74 and 162 SNPs (respectively, the new hits and those found after pooling together different datasets) from the latest GWAS of educational attainment [11] will be employed. Average estimated population IQ will be used as the phenotype of interest and main dependent variable in the analyses. This choice can be justified by its privileged status in psychometric research and its robust genetic correlation ( $r=$ around 0.7 ) with educational performance [12] and attainment [13]. Moreover, the GWAS hits identified by the three educational GWAS also predict general cognitive ability in their samples [9-11]. A re-analysis of the Okbay et al. dataset revealed that the polygenic score also predicts general intelligence (3.6\%) compared to $2 \%$ for the 2013 polygenic score [12].

\section{Materials and Methods}

Rietveld et al. [9] produced 3 SNPs reaching GWAS significance for educational attainment. Davies et al. [10] reported 1115 SNPs reaching GWAS significance, of which 15 were independent signals for educational attainment. 942 SNPs were found on 1000 Genomes. Among the 15 independent signals, one (2:48696432_G_A) was missing.

Okbay et al. [11] reported 74 SNPs associated with years of education. 70 were found in 1000 Genomes (the other 4 variants were flagged because they had more than 3 different alleles). An additional 162 SNPs were reported in the pooled meta-analysis (161 were found in 1000 Genomes).

A simulation was performed using a random dataset, consisting of a large sample $(\mathrm{N}=7369)$ of matched random unlinked SNPs (downloaded from 1000 Genomes, phase 3). Matching was carried out using SNPSNAP[5], by feeding the 9 SNPs and setting LD $r^{2}<0.1$ (for EUR). Fst distances were obtained from [2].Population IQ estimates were obtained from [8]. IQ previously published were used also to guarantee that the values were not created ad hoc.

Analyses were run using $\mathrm{R}[15]$

\section{Results}

A correlation between all the variables (i.e. SNPs frequencies) was run on the entire random dataset (Suppl. File 1). This produced a very large correlation matrix $(\mathrm{N}=27,147,396$ for the lower triangle).

The average correlation coefficient was $0.058(S D=0.537)$. The slightly positive value is likely due to the differential representation of minor alleles among populations. The same analysis was applied to the educational attainment GWAS hits (table 1).

It is clear that there is very little signal in the GWAS hits and it seems to be concentrated within a small subset of SNPs, possibly the 9 replicated loci and the 7 cross-replicated hits ( $r=0.278$ and 0.125 , respectively). Similar null results (table 1) were obtained for the $600+$ SNPs from the largest GWAS of human height [16].

Since there was some LD between the 9 quasi-replicated SNPs, only one SNP per chromosome was retained, yielding 6 unlinked SNPs. This gave a "pure" (LD-free) measure of correlation. The average correlation was slightly higher than for the 9 SNPs $(r=0.343)$, implying that LD did not produce the correlation among the full set of 9 hits.

Table 1. Average intercorrelation between allele frequencies. 


\begin{tabular}{|l|l|l|l|}
\hline & Average r & SD & SE \\
\hline 9 replicated loci & 0.278 & 0.458 & 0.076 \\
\hline $\begin{array}{l}6 \quad \text { replicated loci } \\
\text { (linkage pruned) }\end{array}$ & 0.343 & 0.342 & 0.138 \\
\hline $\begin{array}{l}7 \quad \text { cross-replicated } \\
\text { SNPs }\end{array}$ & 0.125 & 0.455 & 0.117 \\
\hline $\begin{array}{l}\text { 74 EduYears SNPs } \\
\text { (Okbay et al., 2016) }\end{array}$ & 0.003 & 0.496 & 0.011 \\
\hline $\begin{array}{l}161 \quad \text { SNPs, Pooled } \\
\text { meta-analysis [11] }\end{array}$ & 0.004 & 0.495 & 0.004 \\
\hline 691 Height SNPs [16] & 0.006 & 0.514 & 0.006 \\
\hline
\end{tabular}

Correlation between polygenic scores and population IQ

The polygenic score computed using the 9 SNPs was highly correlated $(r=0.9)$ to an estimate [15] of average population IQ (fig. 1). An empirical simulation was run using 819 PS computed from groups of 9 SNPs taken from the random dataset. The average correlation between population IQ and the random polygenic scores was $0.22(\mathrm{~N}=819)$. The slightly positive correlation can be interpreted as an effect of spatial/phylogenetic autocorrelation [8]. Indeed, the correlation between population IQ and the polygenic score of all the random SNPs $(\mathrm{N}=7369)$ was $\mathrm{r}=0.425$, suggesting again the presence of phylogenetic autocorrelation. The increase in the correlation coefficients moving up from low (9)to high SNPs number $(7 \mathrm{k}+)$ is due to the reduction in the noise associated with each SNP. Because the correlation coefficients were not normally distributed (fig. 2), z-score computation was not appropriate. Hence, the percentile corresponding to a correlation coefficient $r=0.9$ was found to be $99.9 \%$ (using the 819 random polygenic scores), implying that the result is highly significant (produced only 1 out of 1000 times using random sets of SNPs).

Figure 1. Correlation between population IQ and polygenic score. 


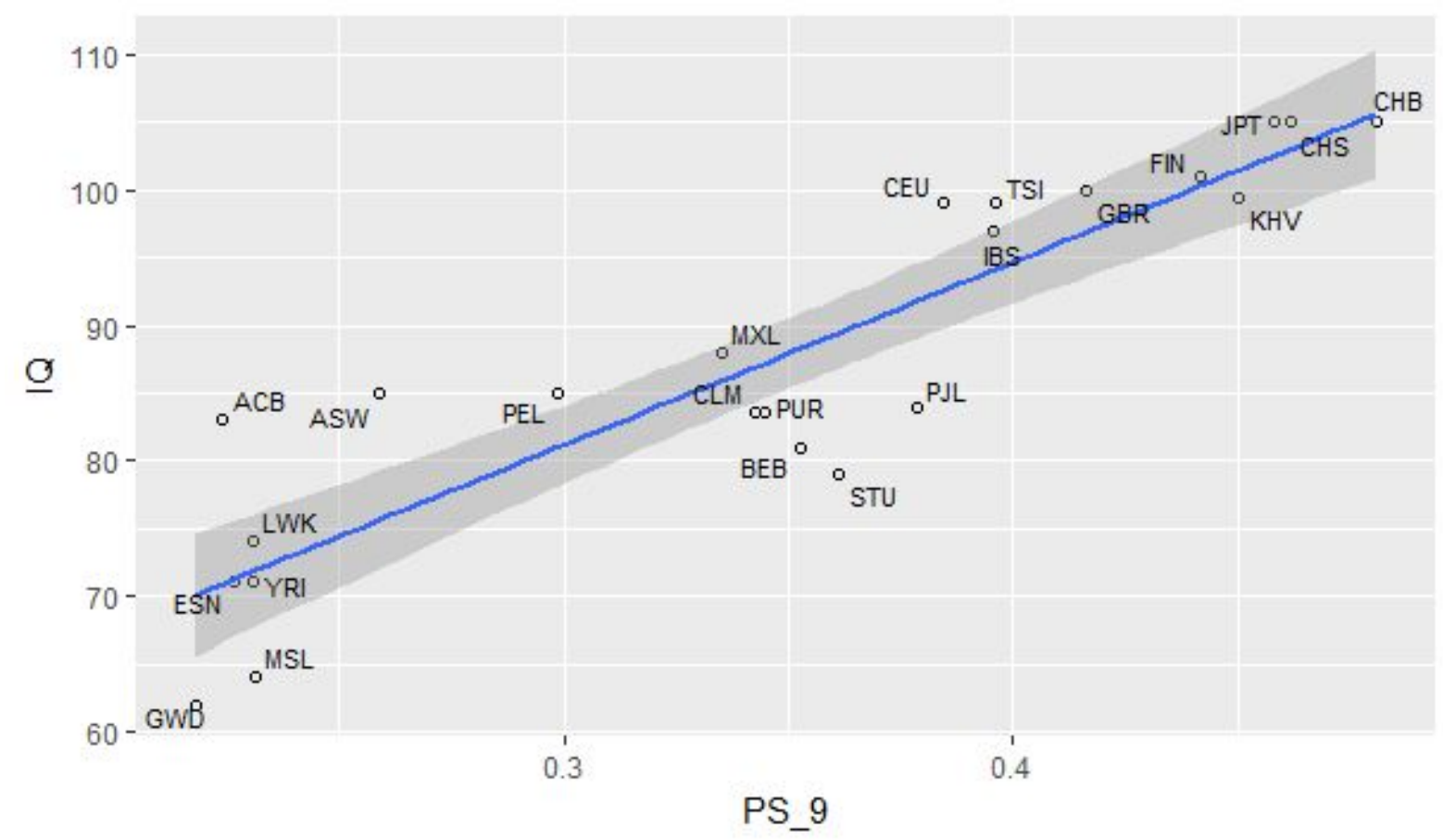

The correlation between the 7 SNPs PS and population IQ was $r=0.832$. The correlation between the 161 SNPs polygenic score and population IQ was high $(r=0.854)$, corresponding to the 95th percentile in the simulation (using PS comprising 161 random SNPs each). The 161 SNPs and the 9 SNPs polygenic scores were strongly correlated ( $r=$ 0.956).

Conversely, the 74 SNPs did not have much predictive power $(r=0.655)$.

Figure 2. Distribution of correlation coefficients ( $r$ population IQ $x$ sets of nine random SNPs).

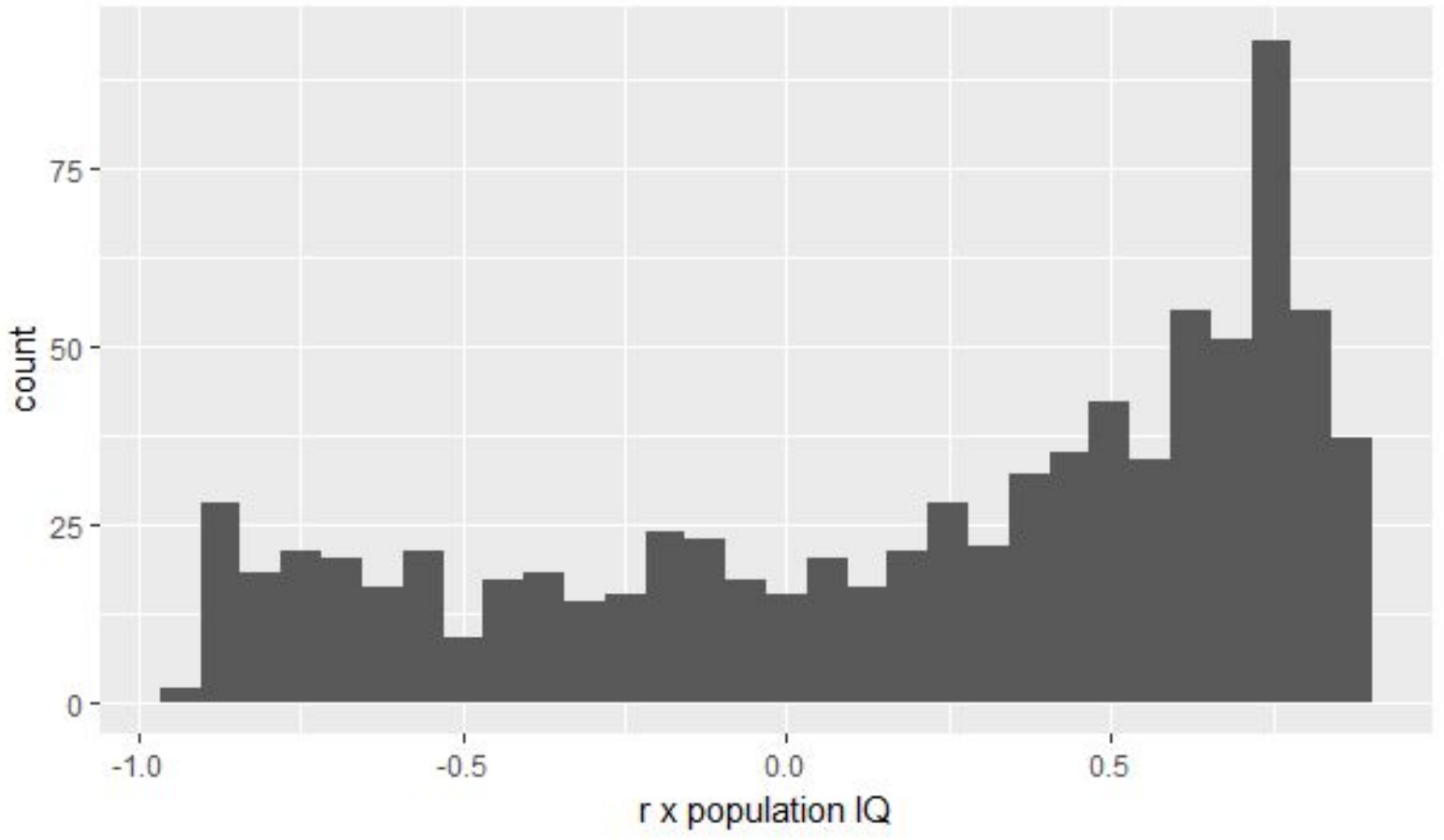




\section{Partialling out spatial autocorrelation using multiple regression}

The presence of spatial autocorrelation in a dataset means that the cases are not independent leading to an overestimation of degrees of freedom and, in the case of positive autocorrelation, an inflation in the correlation between two or more variables. The source of spatial autocorrelation in population genetics datasets is the similarity caused by admixture among neighbouring populations, and the differences caused by random drift. Demonstrating that the alleles predict population-level differences in average phenotypic values above and beyond that predicted on the basis of migration, drift etc, provides evidence for a model of polygenic selection.

Population IQ was regressed on the "random PS" (computed using the $7 \mathrm{k}+$ random SNPs) and the 9 GWAS hits PS. The model was significant ( $F=43.06, p=5.649 e-08$, Adj $R^{2}=0.793$. The random PS had no predictive power $(B=0.037)$, whereas the 9 GWAS hits PS had strong predictive power (Beta $=0.884$ ).

Moreover, Fst distances were also used to partial out spatial autocorrelation, following the method outlined in [1], similar to Mantel test [17]. The correlation between Fst distances and IQ distances was moderate ( $r=0.588$ ), pointing out to the presence of spatial autocorrelation. Multiple regression was performed and a significant model was obtained (Adjusted R-squared: 0.545 , F-statistic: 151.8 on 2 and 250 DF, p-value: $<2.2 \mathrm{e}-16$ ), producing similar effects (table 2).

Table 2. Multiple regression with random SNPs and GWAS hits

\begin{tabular}{|l|l|l|l|l|}
\hline Variable & Beta & $\mathbf{t}$ & sig & VIF \\
\hline $\begin{array}{l}\text { PS 9 GWAS } \\
\text { hits }\end{array}$ & 0.883 & 8.182 & $8.21 \mathrm{e}-08$ & 1.237 \\
\hline Random PS & 0.038 & 0.351 & 0.729 & 1.237 \\
\hline & & & & \\
\hline PS 9 distances & 0.789 & 10.579 & $<2 \mathrm{e}-16$ & 3.082 \\
\hline Fst Distances & -0.061 & -0.806 & 0.421 & 3.082 \\
\hline
\end{tabular}

How does the number of SNPs affect the correlation between average population phenotypic value (i.e. IQ) and polygenic score?

A polygenic score computed from a higher number of SNPs should reduce the noise in the data. In order to test this model, polygenic scores were created using different number of SNPs over the random SNPs dataset. The correlation of each polygenic score with population IQ was computed. The data followed a logarithmic function (figure 3), and the log regression model was compared to a linear model: the former had a much better fit to the 
data (Adjusted R-squared: 0.939, F-statistic: 185.2 on 1 and 11 DF, p-value: 3.16e-08) compared to the latter (F-statistic: 7.568 on 1 and $11 \mathrm{DF}, \mathrm{p}$-value: 0.019 ).

Figure 3. Relationship between number of SNPs and predictive power.

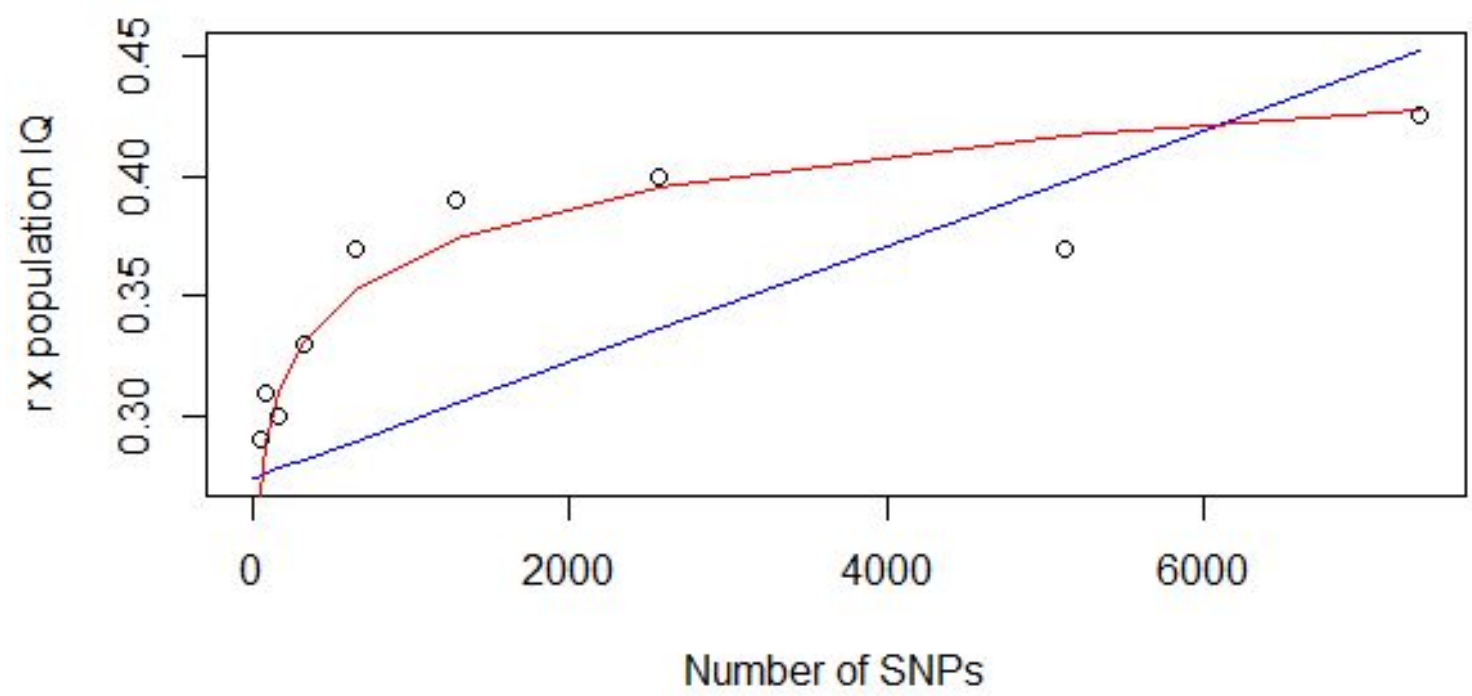

\section{Discussion}

The method of correlating allele frequencies seems to have low power to detect signals of polygenic selection and picks up the signal only for the most powerful genetic loci. Simply computing polygenic scores (average of allele frequencies with positive GWAS beta) seems a more powerful method to detect polygenic adaptation. The polygenic score obtained from 9 quasi-replicated SNPs looks like a good candidate for estimating selection strength on educational attainment, as shown by it outperforming $99.9 \%$ of the polygenic scores obtained from random SNPs and its robustness to tests controlling for spatial autocorrelation (table 2). Despite the lack of internal covariance (table 1) in the larger polygenic score (161 SNPs), its predictive power was strong (outperforming $98 \%$ of the random polygenic scores). The reliability of random polygenic scores increases as a logarithmic function of the number of SNPs, even with random SNPs, hence independently of selection signal and instead due to the reduction in noise. The correlation asymptotically approaches a value around 0.45 (which can be interpreted as the degree of spatial autocorrelation in the dataset). Thus, the predictive power of polygenic scores should to be scaled in proportion to the number of SNPs composing them (i.e. simulations need to be performed using the same number of SNPs).

\section{Appendix}

Supplementary file n.1: SNP freqs.

Supplementary file n.2: Polygenic scores and population IQ 


\section{References:}

1. Piffer, D. (2013). Factor analysis of population allele frequencies as a simple, novel method of detecting signals of recent polygenic selection: The example of educational attainment and IQ. Mankind Quarterly, 54, 168-200.

2. Piffer, D. (2015). A review of intelligence GWAS hits: Their relationship to country IQ and the issue of spatial autocorrelation. Intelligence, 53, 43-50.

3. Pritchard, J. K., Pickrell, J. K., \& Coop, G. (2010). The genetics of human adaptation: hard sweeps, soft sweeps, and polygenic adaptation. Current biology, 20, 208-21

4. Berg, J. J., \& Coop, G. (2014). A population genetic signal of polygenic adaptation. PLoS Genetics, 10, e1004412.

5. Turchin, M. C., Chiang, C. W., Palmer, C. D., Sankararaman, S., Reich, D., Genetic Investigation of, A.T.C, et al. (2012). Evidence of widespread selection on standing variation in Europe at height-associated SNPs. Nature Genetics, 44, 1015-101

6. Zoledwieska et al., 2015. Height-reducing variants and selection for short stature in Sardinia. Nature Genetics 47, 1352-1356

7. Robinson et al. 2015.Population genetic differentiation of height and body mass index across Europe. Nature Genetics, 47,1357-62. doi: 10.1038/ng.3401

8. Piffer, D. Evidence for Recent Polygenic Selection on Educational Attainment Inferred from GWAS Hits. Preprints 2016, 2016110047 (doi: 10.20944/preprints201611.0047.v1).

9. Rietveld, C.A., Medland, S.E., Derringer, J., Yang, J., Esko, T., Martin, N.W., et al. (2013). GWAS of 126,559 individuals identifies genetic variants associated with educational attainment. Science, 340, 1467-1471. doi: http://doi.org/10.1126/science.1235488

10. Davies, G., Armstrong, N., Bis, J. C., Bressler, J., Chourake, V., Giddaluru, S., et al. (2015). Genetic contributions to variation in general cognitive function: a meta-analysis of genome-wide association studies in the CHARGE consortium ( $\mathrm{N}=$ 53949). Molecular Psychiatry, 20, 183-192. http://dx.doi.org/10.1038/mp.2014.188.

11. Okbay, A., Beauchamp, J.P., Fontana, M.A., Lee, J., Pers, T.H., et al. (2016). Genome-wide association study identifies 74 loci associated with educational attainment. Nature, doi:10.1038/nature17671

12. Krapohl, E., Rimfeld, K., Shakeshaft, N.G., Trzaskowski, M., McMillan, A., Pingault, J.-B., Asbury, K., Harlaar, N., Kovas, Y., Dale, P.S. \& Plomin, R. (2014).The high heritability of educational achievement reflects many genetically influenced traits, not just intelligence. PNAS, 111, 15273-15278, doi: 10.1073/pnas.1408777111

13. Bulik-Sullivan, B., Finucane, H. K., Anttila, V., Gusev, A., Day, F. R., Loh, P.-R., ... Neale. (2015). An atlas of genetic correlations across human diseases and traits. Nature Genetics, 47(11), 1236-1241. https://doi.org/10.1038/ng.3406

14. Tune H. Pers, Pascal Timshel, Joel N. Hirschhorn; SNPsnap: a Web-based tool for identification and annotation of matched SNPs. Bioinformatics 2014; 31 (3): 418-420. doi: 10.1093/bioinformatics/btu655 
15. $R$ Core Team (2016). R: A language and environment for statistical computing. $R$ Foundation for Statistical Computing, Vienna, Austria. URL: https://www.R-project.org/.

16. Wood AR, Esko T, Yang J, et al:: Defining the role of common variation in the genomic and biological architecture of adult human height. Nat Genet. 2014; 46(11): 1173-86

17. Mantel, N. (1967). The detection of disease clustering and a generalized regression approach. Cancer Research. 27, 209-220.

Table S1. Pseudo-replicated and replicated SNPs. Sites in LD $(r>0.8)$.

\begin{tabular}{|l|l|l|l|l|l|}
\hline Publication & Index SNP & Publication & Linked SNP & D' & $\mathbf{r}^{2}$ \\
\hline $\begin{array}{l}\text { Davies et al., } \\
2016\end{array}$ & $\begin{array}{l}\text { rs12042107_ } \\
\text { C }\end{array}$ & $\begin{array}{l}\text { rs1008078_ } \\
\text { C }\end{array}$ & $\begin{array}{l}\text { Okbay et al., } \\
2016\end{array}$ & 1 & 0.8 \\
\hline $\begin{array}{l}\text { Rietveld et } \\
\text { al., } 2013\end{array}$ & $\begin{array}{l}\text { rs11584700_ } \\
\text { G }\end{array}$ & $\begin{array}{l}\text { rs1158885___ } \\
\text { A }\end{array}$ & $\begin{array}{l}\text { Okbay et al., } \\
2016\end{array}$ & 1 & 0.94 \\
\hline $\begin{array}{l}\text { Rietveld et } \\
\text { al., 2013 }\end{array}$ & $\begin{array}{l}\text { rs4851266_ } \\
\text { T }\end{array}$ & $\begin{array}{l}\text { rs12987662_ } \\
\text { A }\end{array}$ & $\begin{array}{l}\text { Okbay et al., } \\
2016\end{array}$ & 1 & 1 \\
\hline $\begin{array}{l}\text { Davies et al., } \\
2016\end{array}$ & $\begin{array}{l}\text { rs13086611_ } \\
\text { T }\end{array}$ & $\begin{array}{l}\text { rs148734725 } \\
\text { A }\end{array}$ & $\begin{array}{l}\text { Okbay et al., } \\
2016\end{array}$ & 1 & 1 \\
\hline $\begin{array}{l}\text { Davies et al., } \\
2016\end{array}$ & $\begin{array}{l}\text { rs11130222_ } \\
\text { A }\end{array}$ & $\begin{array}{l}\text { rs11712056_ } \\
\text { T }\end{array}$ & $\begin{array}{l}\text { Okbay et al., } \\
2016\end{array}$ & 1 & 0.98 \\
\hline $\begin{array}{l}\text { Davies et al., } \\
2016\end{array}$ & $\begin{array}{l}\text { rs55686445_ } \\
\text { C }\end{array}$ & $\begin{array}{l}\text { rs62263923_ } \\
\text { G }\end{array}$ & $\begin{array}{l}\text { Okbay et al., } \\
2016\end{array}$ & 1 & 0.98 \\
\hline $\begin{array}{l}\text { Davies et al., } \\
2016\end{array}$ & $\begin{array}{l}\text { rs12553324_ } \\
\text { G }\end{array}$ & $\begin{array}{l}\text { rs13294439_ } \\
\text { C }\end{array}$ & $\begin{array}{l}\text { Okbay et al., } \\
2016\end{array}$ & 1 & 0.98 \\
\hline $\begin{array}{l}\text { Davies et al., } \\
2016\end{array}$ & $\begin{array}{l}\text { rs4799950_ } \\
\text { G }\end{array}$ & $\begin{array}{l}\text { rs12969294_ } \\
\text { G }\end{array}$ & $\begin{array}{l}\text { Okbay et al., } \\
2016\end{array}$ & 1 & 0.92 \\
\hline $\begin{array}{l}\text { Rietveld } 2013 \\
\text { A et }\end{array}$ & As9320913_ & $\begin{array}{l}\text { Okbay et al., } \\
2016\end{array}$ & 1 & 1 \\
\hline
\end{tabular}

